\title{
Secure Peer Review and Feedback on Medical Findings via IHE XDS
}

\author{
Emmanuel HELM ${ }^{\mathrm{a}, 1}$, Jacqueline SCHWEBACH ${ }^{\mathrm{a}, \mathrm{b}}$, Andreas POINTNER ${ }^{\mathrm{a}}$, Anna LIN ${ }^{\mathrm{a}}$, \\ Valentin ROTHENSTEINER ${ }^{\mathrm{b}}$, Dietmar KEIMEL ${ }^{\mathrm{b}}$ and Andreas SCHULER $^{\mathrm{a}}$ \\ ${ }^{a}$ University of Applied Sciences Upper Austria, Research Department for \\ Advanced Information Systems and Technology, 4232 Hagenberg, Austria \\ ${ }^{b}$ CAS - Computer Anwendungs Systeme GmbH., 1090 Wien, Säulengasse 6/9
}

\begin{abstract}
Background: There is a lack of secure official communication channels for peer review and peer feedback on medical findings. Objectives: We aimed to utilize the existing Austrian eHealth infrastructure to enable review and feedback processes. Methods: We extended the IHE XDW workflow document to enable the exchange of text messages (i.e., comments on documents or images) over an XDS infrastructure. Results: The workflow enables the exchange of comments on specific sections of CDA documents or radiological images and was verified in an XDS test environment. Conclusion: The presented solution is a proof of concept and the potential basis for the specification of a new IHE workflow definition.
\end{abstract}

Keywords. Workflow [L01.906.893], Radiology [H02.403.740]

\section{Introduction}

We developed a system for discussing findings and obtaining expert opinions and feedback via secure medical networks based on the Integrating the Healthcare Enterprise (IHE) Cross-Enterprise Document Sharing (XDS) integration profile. In this paper we describe the approach and discuss the results.

In 2018, Helm et al. [1] published a study on cross-enterprise communication and data exchange in Austrian radiology practices and departments. They conducted interviews with 4 experts each from hospitals and practices to identify the use cases and technology used for communication and data exchange. A key finding was that radiologists mainly use informal channels to obtain expert opinions, peer reviews, and peer feedback, e.g., online messengers [1].

This leads to several issues, e.g.: (1) under data protection law, the exchange of patient information via, e.g., WhatsApp is questionable, (2) the resolution of photos in these messenger apps is not suitable for a qualified assessment of radiological images, and (3) the communication is not available for follow-up visits and other physicians.

Thus, our motivation was to provide the means to enable secure communication of findings, expert opinions, and feedback over the existing Austrian eHealth infrastructure. To this end, we utilize the standards-based, centrally available services of the Austrian national electronic health record ELGA (Elektronische Gesundheitsakte) [2].

\footnotetext{
Corresponding Author: Emmanuel Helm, FH OÖ F\&E GmbH, Hagenberg, Austria, E-Mail: emmanuel.helm@fh-hagenberg.at
} 


\subsection{Background}

The Austrian national electronic health record ELGA enables patient-centric document sharing across different healthcare providers. The architecture is based on the interplay of actors and transactions specified in the integration profiles of the non-profit organization IHE. These integration profiles define how to utilize established standards like Health Level Seven (HL7) or Digital Imaging and Communications in Medicine (DICOM) to solve common interoperability problems, e.g., the document exchange between healthcare providers [3]. In ELGA, the structure of the documents is based on the HL7 Clinical Document Architecture (CDA) standard.

In simplified terms, three transactions of the IHE XDS integration profile [4] are relevant to exchange clinical reports: (1) ITI-18 Registry Stored Query, to receive the metadata for a set of documents of a given patient, (2) ITI-41 Provide and Register Set$b$, to upload a document, and (3) ITI-43 Retrieve Document Set, to receive a document. For the exchange of medical images, the Cross-enterprise Document Sharing for Imaging (XDS-I) integration profile [2], an extension of XDS, is relevant.

The IHE Cross-Enterprise Document Workflow (XDW) integration profile [4] states: "This profile defines an instrument, called a 'Workflow Document', to manage and track a shared workflow. It records the creation of tasks and maintains a historical record of tasks as they move through the associated workflow". While XDW defines form and function of this document, the actual sharing of the document is based on XDS. And while XDW is not part of ELGA yet, the existing XDS infrastructure already provides the means for future adoption of this integration profile.

\subsection{Problem Statement}

Previous research $[1,5,6]$ described a lack of official communication channels for obtaining external feedback or expert opinions on reports or radiological imaging. In Austria, mainly informal channels are used, e.g., telephone exchanges with acquainted physicians or the use of online messengers with other peers [1]. In addition to quality, privacy, and security issues with online messengers, the lack of official channels for exchange makes business models for proven experts impossible. Currently, ELGA lacks active workflow support. To overcome these problems, we developed a workflow mechanism that enables cross-enterprise collaboration based on the existing eHealth infrastructure. We show its applicability in the radiology domain.

\subsection{Relevance and Related Work}

In 2014, Austria introduced the nationwide peer review program "Früh erkennen" [7] for breast cancer screening. To reduce the number of false negative and false positive diagnoses, radiologists are obliged to obtain a second opinion on their Breast Imaging Reporting And Data System (BIRADS) assessment. Strickland [5] describes the potential for quality assurance in radiology via the introduction of peer review and peer feedback processes. However, he also describes a lack of integrated system software on the market. Gunn et al. [6] point out existing tools such as RADPEER (ACR) but also highlight problems of sole radiologist-to-radiologist peer review (e.g., lack of context, or practices and terminology confusing to end-readers). They found that including the referring physician in the process is a potential solution to overcome these problems. 


\section{Methods}

To enable peer review and peer feedback processes (i.e., workflows) on the existing Austrian eHealth infrastructure, we extended the XDW Workflow Document [4] with an "annotation" mechanism. The workflow document basically contains a structured list of tasks, documenting the execution of a workflow. In our case, a peer review or peer feedback process constitutes a workflow document, with a task for the communication of the peers. The workflow document itself is exchanged via the XDS infrastructure, as is the respective report that is referred to.

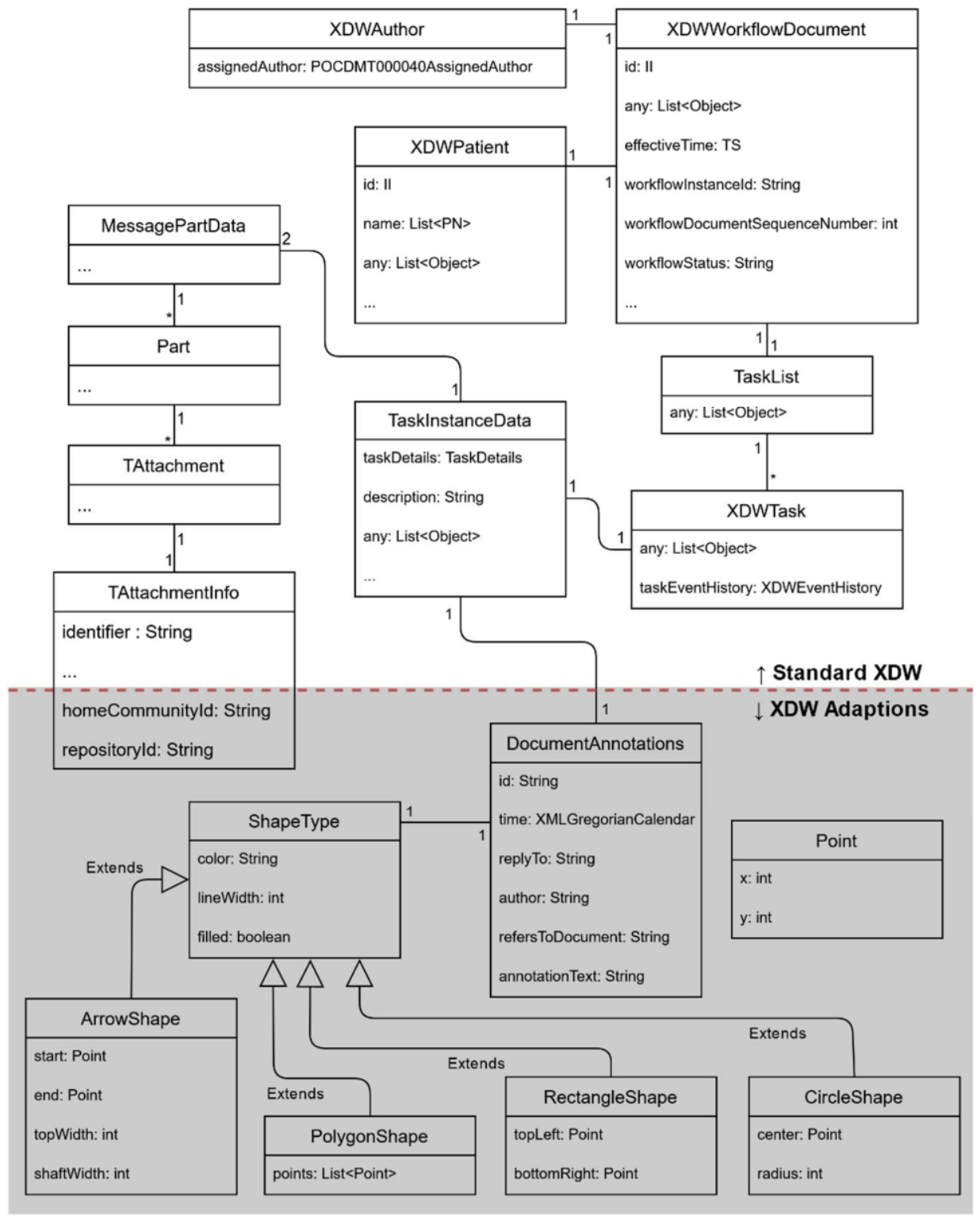

Figure 1: Schema outlining the adaptions to the XDW Document. 


\subsection{Adding Comments to XDW Workflow Documents}

To utilize the XDW workflow document for comments, it was necessary to perform some adaptions (see Figure 1). We added objects that allow to write comments for specific parts of a referenced document or image. A documentAnnotation element was added to fulfill that requirement. This class contains the basic information required for a comment, namely an author, a date (time), and an annotationText. In addition, an ID to uniquely identify a comment inside an XDW document as well as a replyTo property - allowing to reply to specific comments - were added. For the rest of the adaptions, we must differentiate between commenting on CDA documents or DICOM objects (i.e., images).

For CDA documents it should be possible to comment on specific sections. For this purpose, a refersToDocument field was added, allowing to reference a section of a CDA document (cf. Listing 1). Furthermore, it is necessary to distinguish between top level comments and replies. For each top-level comment, the children are attached recursively matching the comment ID and the replyTo property.

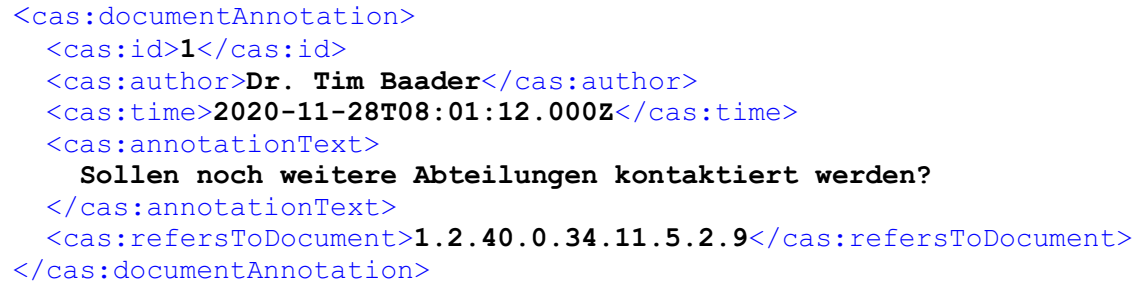

Listing 1: Referring to a CDA-Section in an XDW-Document using documentAnnotation.

In case of DICOM, or more generally in the case of images, it should be possible to refer to an explicit position in the picture. We developed a concept to allow adding shapes with annotations as seen in Listing 2. For that purpose, Markers were added to the DocumentAnnotation. Each marker consists of a specific shape, e.g., Arrow, Polygon, Rectangle or Circle. These shapes do have some properties in common, i.e., a specific color, a line width, and a flag that indicates if the shape is filled or frame only. In addition to that, each shape type has properties to define its position, size, and form.

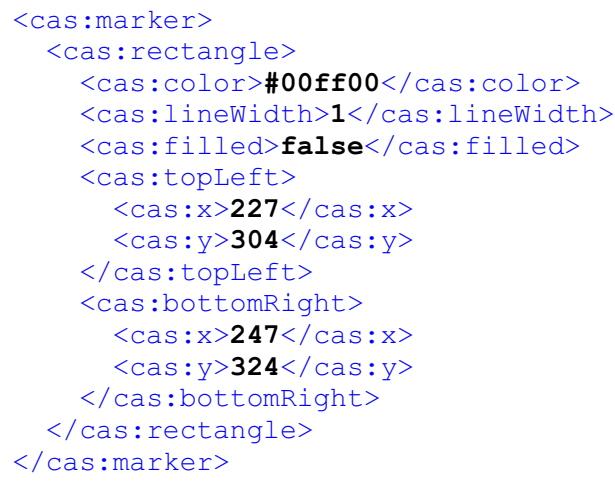

Listing 2: Description of an image marker inside a documentAnnotation. 


\subsection{Referencing Reports or Images}

An XDW document describes multiple tasks, where each task with its TaskInstanceData can define input and output to refer to relevant objects for the task (e.g., diagnostic reports). In our case, one XDW workflow document describes the workflow of a peer review or peer feedback to a single CDA document or DICOM object. The input of the task in the XDW document refers to the respective document or object.

However, the standard XDW input and output fields do not have all the information needed for retrieval of the object in a cross-community XDS environment (based on the XCA profile [4], e.g., ELGA [2]) via the IHE ITI-43 Retrieve Document Set transaction. This transaction needs at least a sourceDocumentId, a homecommunityId as well as a repositoryId [2]. To add those fields, we extended the AttachmentInfo class, by adding fields for the homecommunityId and repositoryId. The identifier field of the object was used to store the sourceDocumentId.

To retrieve DICOM objects, the IHE RAD-69 Retrieve Imaging Document Set was used as specified by [8]. Thus, Study-, Series-, Instance- and HomeCommunityId is required. These fields are again added as extensions to the AttachmentInfo class.

\subsection{Testing Infrastructure}

ELGA provides a test environment, which we used as a testing infrastructure. To avoid implementing the required SOAP transactions, we used Open eHealth Integration Platform (IPF) components [9]. These components provide an abstraction for accessing the IHE-based ELGA transaction endpoints. By using IPF, we can exchange CDA documents and image references via the ELGA test registries and repositories. In theory this would also enable us to upload XDW workflow documents, but by the time of this work this was not supported by the ELGA infrastructure.

For testing the XDW workflow, a custom XDW document store was implemented. A file storage providing a basic REST interface was hosted on our test server. This endpoint provides methods to query and store XDW workflow documents. In addition to that a simple client was developed, that allows to display CDA documents as well as images and provides the means to add comments via the described approach.

\section{Results}

The presented approach allows to comment on specific sections of CDA documents and images to enable the collaboration across different departments or even enterprises. The following results show the communication in an example client application. We were able to test and simulate different scenarios on the ELGA-based testing infrastructure and provide two examples here.

The comment section has the same functionality and design for both scenarios. It shows the different comments, that are either assigned to a specific section for CDA documents or to a specific region in the image. Each comment consists of three parts: (1) the author, (2) a date when the comment was submitted, and (3) the comment text. There are two different types of comments: Top-level comments that belong to a specific document section or image region, and answer comments, that reply on another comment. They can be distinguished by their indentations (cf. comment section in Figures 2 and 3). 


\subsection{Comments on Reports}

The first example, cf. Figure 2, shows the communication between two physicians in a finding represented by a CDA document. The image is split into two sections. On the left-hand side, a rendered form of the CDA document is displayed, whereas the righthand side shows the comment section. To provide a uniform representation across platforms, ELGA [2] defines a default layout for CDA documents and provides the respective Extensible Stylesheet Language Transformation (XSLT) file. This stylesheet can be applied on the XML representation of the CDA document, and transforms it into the HTML format, which can be displayed as shown in our example.

In addition to that, we added the comment concept. This consists of two parts, on the one hand there are the comments with the authors on the right side, on the other hand there is a circle with number in it for every specific section in the CDA document (cf. Figure 2). These numbers represent the comments that are written in a specific section.

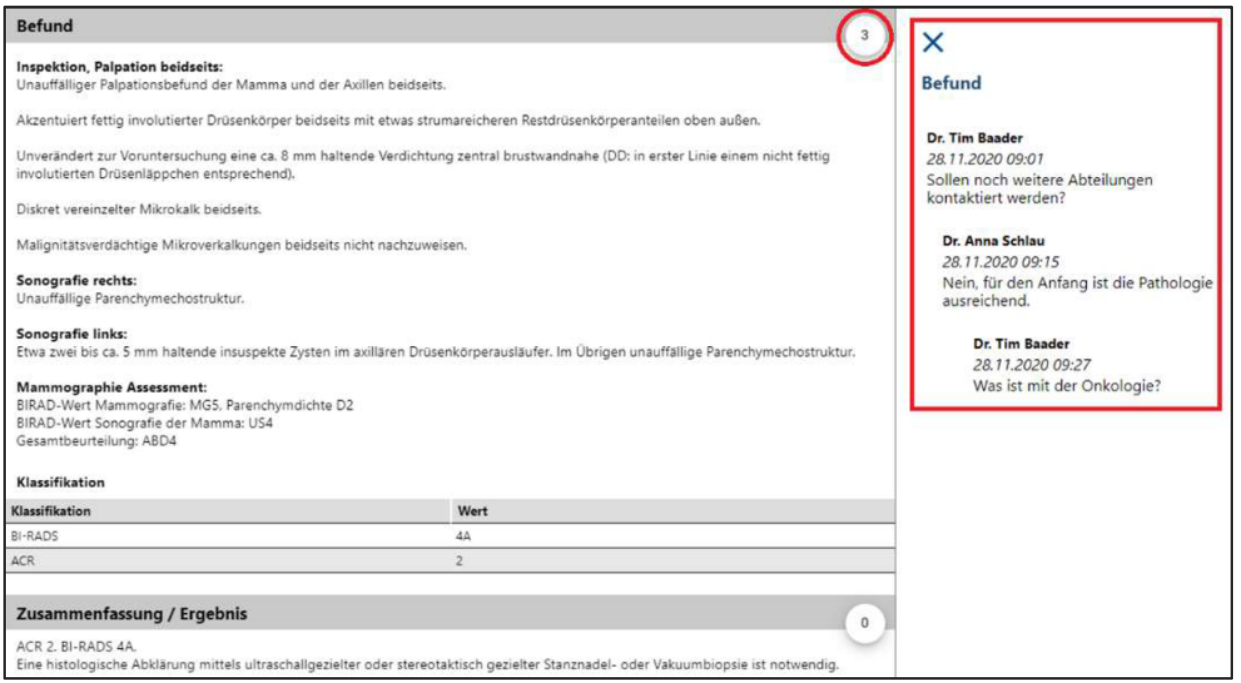

Figure 2: CDA document rendered with the ELGA stylesheet (left). The red circle highlights the number of comments in this specific section. The comments are visible on the right side.

\subsection{Comments on Images}

The second example, cf. Figure 3, shows the communication between two physicians but in this case for a single image. The left side shows the menu bar on top as well as the image, whereas on the right side the comments are placed. We utilize the RAD-69 transaction to query for a rendered JPEG image using the respective Transfer Sytax property. The image can be annotated with different forms, e.g., circles or arrows. Each of the shapes can have a different colour as well as different line width. In addition to the comments section on the right, each top-level comment is extended with the used shape as well as the colour to highlight the connection. Each new shape automatically creates a new top-level comment as well. 


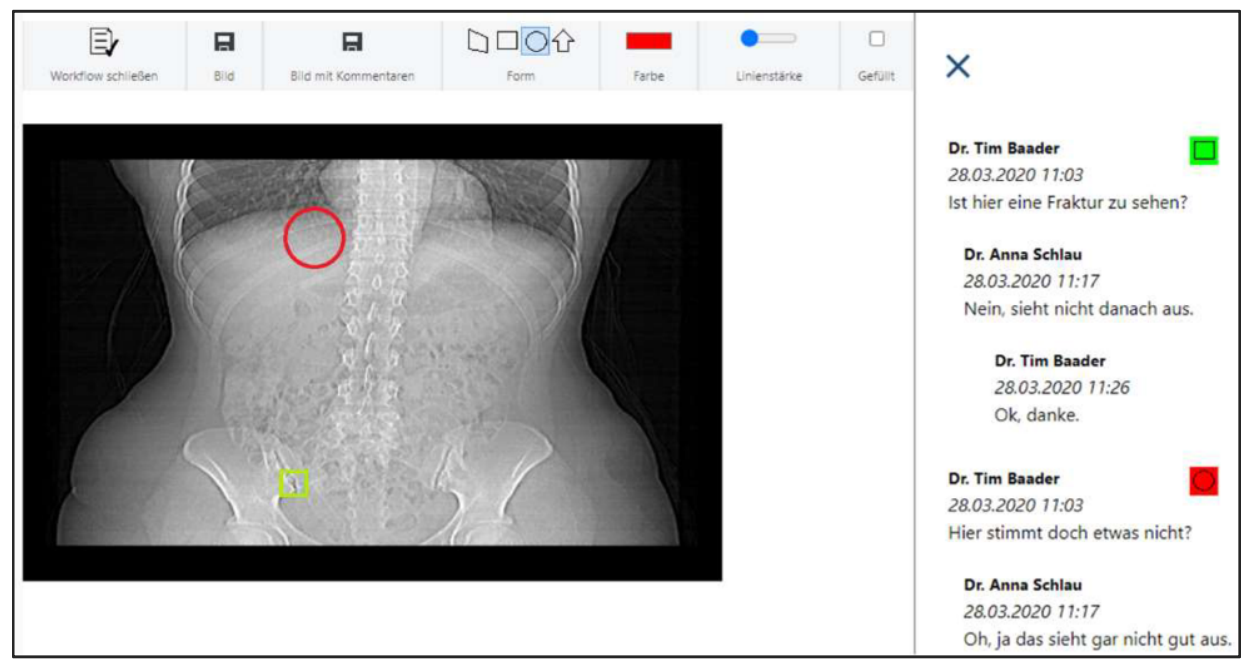

Figure 3: A radiology image with annotating shapes in the image and respective comments on the right side.

\section{Discussion}

The presented work is a first proof of concept on how to utilize XDW workflow documents for establishing secure and integrated communication channels in context of medical reports and images. There are still open issues and threats to the validity of the presented approach:

1. There is no IHE workflow definition document describing the use of XDW workflow documents for this purpose. Thus, the presented approach can only be seen as a predecessor of future standardized solutions.

2. ELGA does not support XDW workflow documents. These documents cannot be exchanged via the Austrian eHealth infrastructure (yet). However, the approach could already be implemented in local (XDS) environments.

3. The image approach only works on single rendered JPEGs. We did not implement a full DICOM viewer. However, the concept to store comments on DICOM objects in XDW workflow documents and exchange them via an XDS/ XCA infrastructure could be of interest for DICOM viewer or PACS vendors.

4. The described approach does not allow to address a specific peer with a request for feedback or expert opinion. Currently, the XDW document with the questions and comments, linked to a patient's CDA document or DICOM image, would be available to all healthcare providers with a valid treatment assertion for the patient. Thus, the approach uses ELGA as a platform, but would require additional peer-to-peer functionality for direct communication.

5. Due to the document-based nature of this approach, every exchange of comments or annotations requires the retrieval of the complete XDW workflow document. This leads to a certain redundancy in the communication. However, the XDW workflow document does not require significant bandwidth, e.g., the document in the examples above has $5 \mathrm{kB}$ with about 100 lines of code. 


\section{Conclusions and Outlook}

Our motivation was to provide the means to enable secure and integrated communication of findings, expert opinions, and feedback over the existing eHealth infrastructure. We presented an approach to utilize XDW workflow documents to enable cross-enterprise peer review and peer feedback. The approach was developed with respect to the Austrian eHealth infrastructure ELGA $[2,8]$ and is mostly based on international standards.

In practice, the developed workflow is either document centric or image centric. Comments can be made on sections of documents if they are structured based on the HL7 CDA standard. Alternatively, regions in images can be highlighted and commented.

In IHE the concept of a workflow definition can be used to specify how an XDW workflow document should be utilized to enable a certain workflow. Common examples are the Cross-enterprise Tumor Board Workflow Definition (XTB-WD) and the Crossenterprise Basic eReferral Workflow Definition (XBeR-WD). Given the interest of the Austrian eHealth community, we plan to take a similar approach and prepare a first draft for a Cross-Enterprise (or even Cross-Community) Peer Review Workflow Definition (e.g., XPR-WD) document. Of course, this effort must be coordinated with multiple stakeholders, including the different chapters in IHE.

\section{References}

[1] E. Helm, A. Schuler and H. Mayr, "Cross-Enterprise Communication and Data Exchange in Radiology in Austria: Technology and Use Cases”, Studies in Health Technology and Informatics, vol. 248, pp. 64-71, 2018

[2] ELGA GmbH, "ELGA-Gesamtarchitektur", [Online]. Version 2.30b, Available: https://www.elga.gv.at/ fileadmin/user_upload/Dokumente_PDF_MP4/Technisches/ELGA_Gesamtarchitektur_2.30b.pdf, 2017, [Last Accessed 18.01.2021].

[3] T. Benson und G. Grieve, Principles of Health Interoperability, Springer, 2016.

[4] IHE International, Inc., "IHE IT Infrastructure (ITI) Technical Framework", vol. 1 (ITI TF-1) Integration Profiles, rev. 17.0, July 20, 2020

[5] N. H. Strickland, "Quality assurance in radiology: peer review and peer feedback", Clinical radiology, 70(11), pp. 1158-1164, 2015

[6] A. J. Gunn, C. I. Alabre, S. E. Bennett, M. Kautzky, T. Krakower, K. Palamara und G. Choy, "Structured feedback from referring physicians: a novel approach to quality improvement in radiology reporting", American Journal of Roentgenology, 201(4), pp. 853-857, 2013.

[7] K. Jeitler, T. Semlitsch, N. Posch, A. Siebenhofer und K. Horvath, "Breast cancer screening in Austria: Key figures, age limits, screening intervals and evidence", Zeitschrift für Evidenz, Fortbildung und Qualität im Gesundheitswesen, Bd. 109, Nr. 4, pp. 363-370, 2015

[8] ELGA GmbH, " Anbindung von DICOM Ressourcen in ELGA“", [Online]. Version 1.65, Available: https:// www.elga.gv.at/fileadmin/user_upload/Dokumente_PDF_MP4/Technisches/AnbindungBilddaten_Ges amtarchitektur.pdf, 2020, [Last Accessed 18.01.2021].

[9] Open eHealth Foundation, "Open eHealth Integration Platform - IPF", [Online]. Available: https://github.com/oehf/ipf. [Last Accessed 18.01.2021]. 